\title{
ON SOME SUBCLASSES OF BI-PSEUDO-STARLIKE FUNCTIONS DEFINED BY SĂLǍGEAN DIFFERENTIAL OPERATOR
}

\author{
TIMILEHIN GIDEON SHABA \\ Department of Mathematics, University of Ilorin, Ilorin, Nigeria \\ Corresponding author: shabatimilehin@gmail.com
}

Received Nov. 7, 2020

\begin{abstract}
AвsтRACт. By applying Sălăgean operator, two new subclasses of bi-univalent functions associated with pseudo-starlike function in $\nabla$ which are denoted by $\mathfrak{B}_{\mathfrak{E}}^{\phi, b}(\beta, \psi)$ and $\mathfrak{B}_{\mathfrak{E}}^{\phi, b}(\mu, \psi)$. Also, we investigated estimates on the coefficients $\left|m_{2}\right|$ and $\left|m_{3}\right|$ for functions in these new subclasses and significance of the results are indicated.
\end{abstract}

2010 Mathematics Subject Classification. 30C45; 30C50.

Key words and phrases. Bi-univalent; univalent; Sălăgean operator; pseudo-starlike functions; coeffients bounds.

\section{INTRODUCTION}

Let $\mathfrak{A}$ be the class of analytic function $f(z)$ in the open unit disk $\nabla=\{z: z \in \mathcal{C}:|z|<1\}$, which is normalized by the conditions $f^{\prime}(0)=1$ and $f(0)=0$ of the form

$$
f(z)=z+\sum_{u=2}^{\infty} m_{u} z^{u}
$$

Further, let $\mathcal{S} \subset \mathfrak{A}$ which are univalent functions in $\nabla$. let $S^{*}(\alpha)$ and $\mathcal{K}(\alpha)$ indicate the well known classes of starlike and convex functions of order $\alpha(0 \leq \alpha<1)$ respectively (see [6]). Let $f^{-1}(z)$ be the inverse of the function $f(z)$ then we have

$$
f^{-1}(f(z))=z
$$

and

$$
f\left(f^{-1}(h)\right)=h, \quad|h|<r_{0}(f) ; r_{0}(f) \geq \frac{1}{4}
$$

DOI: 10.28924/APJM/8-6 
where

$$
g(h)=f^{-1}(h)=h-m_{2} h^{2}+\left(2 m_{2}^{2}-m_{3}\right) h^{3}-\left(5 m_{2}^{3}-5 m_{2} m_{3}+m_{4}\right) h^{4}+\cdots
$$

A function $f(z) \in \mathfrak{A}$ is said to be bi-univalent in $\nabla$ if both $f(z)$ and $f^{-1}(z)$ are univalent in $\nabla$. The class of analytic bi-univalent function in $\nabla$ is denoted by $\mathfrak{E}$.

Examples of functions in the class $\mathfrak{E}$ are

$$
-\log (1-z), \quad \frac{z}{1-z}, \quad \frac{1}{2} \log \left(\frac{1+z}{1-z}\right)
$$

and so on. However, the familiar Koebe function is not a member of $\mathfrak{E}$. Other common examples of functions in $\mathcal{S}$ such as

$$
\frac{z}{1-z^{2}} \quad \text { and } \quad z-\frac{z^{2}}{2}
$$

are also not membars of $\mathfrak{E}($ see $[7,12])$.

Lewin [10] (1967) investigated the bi-univalent function class $\mathfrak{E}$ and showed that $\left|a_{2}\right|<1.51$. Subsequently, Brannan and Clunie [3] conjectured that $\left|a_{2}\right| \leq \sqrt{2}$. Netanyahu [12], on the other hand, showed that $\left|a_{2}\right| \leq \frac{4}{3}$. Brannan and Taha [5] (see also [21]) introduced certain subclasses of the bi-univalent function class $\mathfrak{E}$ similar to the familiar subclasses $S^{*}(\beta)$ and $\mathcal{K}(\beta)$ of starlike and convex functions of order $\beta(0 \leq \beta<1)$, respectively (see [4]). Thus, following Brannan and Taha [5], a function $f \in \mathfrak{A}$ is in the class $S_{\mathfrak{E}}^{*}(\beta)$ of strongly bi-starlike of order $\beta(0<\beta \leq 1)$, if

$$
f \in \mathfrak{E}, \quad\left|\arg \left(\frac{z f^{\prime}(z)}{f(z)}\right)\right|<\frac{\beta \pi}{2}, z \in \nabla ; \quad 0<\beta \leq 1
$$

and

$$
\left|\arg \left(\frac{h g^{\prime}(h)}{g(h)}\right)\right|<\frac{\beta \pi}{2}, h \in \nabla ; 0<\beta \leq 1,
$$

where the function $g$ is given by (1.2).

Similarly, a function $f \in \mathfrak{A}$ is in the class $\mathcal{K}_{\mathfrak{E}}(\beta)$ of strongly bi-convex functions of order $\beta(0<\beta \leq 1)$ if

$$
f \in \mathfrak{E}, \quad\left|\arg \left(1+\frac{z f^{\prime \prime}(z)}{f^{\prime}(z)}\right)\right|<\frac{\beta \pi}{2}, z \in \nabla ; \quad 0<\beta \leq 1
$$

and

$$
\left|\arg \left(1+\frac{h g^{\prime \prime}(h)}{g^{\prime}(h)}\right)\right|<\frac{\beta \pi}{2}, h \in \nabla ; \quad 0<\beta \leq 1,
$$

where the function $g$ is given by (1.2). 
The classes $S_{\mathfrak{E}}^{*}(\alpha)$ and $\mathcal{K}_{\mathfrak{E}}(\alpha)$ of bi-starlike functions of order $\alpha$ and bi-convex functions of order $\alpha$, corresponding (respectively) to the function classes $S^{*}(\alpha)$ and $\mathcal{K}(\alpha)$ were also introduced analogously. For each of the function classes $S_{\mathfrak{E}}^{*}(\beta)$ and $\mathcal{K}_{\mathfrak{E}}(\beta)$, Brannan and Taha [5] found non-sharp estimates on the first two Taylor-Maclaurin coefficient $\left|a_{2}\right|$ and $\left|a_{3}\right|$. But the coefficient estimates problem for each of the following Taylor-Maclaurin coefficients $\left|a_{n}\right|$ for $n \in \mathcal{N} \backslash\{1,2\} ; \mathcal{N}:=\{1,2,3, \cdots\}$ is presumably still an open problem. More details about certain subclasses of the bi-univalent function class $\mathfrak{E}$ see [10], [21], [18], [3], [1], [11], [13], [22], [12], [14], [17], [19].

These Functions and its various generalizations have large number of applications in problems of physical sciences, geometry and geometric function theory (for details see [20]). In [2] Babalola defined the class of $\phi$-pseudo starlike functions of order $\psi$ and prove that all Pseudo-starlike functions are Bazelivic of type $\left(1-\frac{1}{\phi}\right)$, order $\psi^{\frac{1}{\phi}}$ are univalent in $\nabla$. For $f(z) \in \mathfrak{A}$, Salagean [16] introduced the differential operator $\mathfrak{D}^{b}$ which is defined by

$$
\begin{gathered}
\mathfrak{D}^{0} f(z)=f(z) ; \\
\mathfrak{D}^{1} f(z)=\mathfrak{D} f(z)=z f^{\prime}(z) ; \\
\mathfrak{D}^{b} f(z)=\mathfrak{D}\left(\mathfrak{D}^{b-1} f(z)\right), \quad b \in \mathcal{N}=1,2,3, \cdots,
\end{gathered}
$$

then,

$$
\mathfrak{D}^{b} f(z)=z+\sum_{u=2}^{\infty} u^{b} m_{u} z^{u}
$$

where $b \in \mathcal{N}_{0}=\mathcal{N} \cup\{0\}=0,1,2,3, \cdots$.

In this present paper, inspired by the earlier work of Babalola [2] and Joshi et. al. [8], we introduce the subclasses $\mathfrak{B}_{\mathfrak{E}}^{\phi, b}(\beta, \psi)$ and $\mathfrak{B}_{\mathfrak{E}}^{\phi, b}(\mu, \psi)$ of the function class $\mathfrak{E}$ associated with Salagean differential operator and determine the bounds on the initial coefficients $\left|m_{2}\right|$ and $\left|m_{3}\right|$. We need the following Lemma in other to establish our main results.

Lemma 1.1. [15] If $r(z) \in \mathcal{P}$ and $z \in \nabla$, then $\left|w_{n}\right| \leq 2$ for each $n$. where $\mathcal{P}$ is the family of all function $u$ analytic in $\nabla$ for which $\Re(r(z))>0$,

$$
r(z)=1+w_{1} z+w_{2} z^{2}+\cdots
$$


2. Coefficient Bounds For the Function Class $\mathfrak{B}_{\mathfrak{E}}^{\phi, b}(\beta, \psi)$

Definition 2.1. A function $f(z)$ given by (1.1) is said to be in the class $\mathfrak{B}_{\mathfrak{E}}^{\phi, b}(\beta, \psi)$ if the following conditions are satisfied:

$$
\left|\arg \left[\frac{z\left[\left(\mathfrak{D}^{b} f(z)\right)^{\prime}\right]^{\phi}}{(1-\psi) \mathfrak{D}^{b} f(z)+\psi \mathfrak{D}^{b+1} f(z)}\right]\right|<\frac{\beta \pi}{2} \quad z \in \nabla,
$$

and

$$
\left|\arg \left[\frac{h\left[\left(\mathfrak{D}^{b} g(h)\right)^{\prime}\right]^{\phi}}{(1-\psi) \mathfrak{D}^{b} g(h)+\psi \mathfrak{D}^{b+1} g(h)}\right]\right|<\frac{\beta \pi}{2} \quad h \in \nabla,
$$

where $f(z) \in \mathfrak{E}, \phi \geq 1,0<\beta \leq 1,0 \leq \psi<1$ and

$$
g(h)=h-m_{2} h^{2}+\left(2 m_{2}^{2}-m_{3}\right) h^{3}-\left(5 m_{2}^{3}-5 m_{2} m_{3}+m_{4}\right) h^{4}+\cdots
$$

Remark 2.1. Taking $\psi=0$ in the class $\mathfrak{B}_{\mathfrak{E}}^{\phi, b}(\beta, \psi)$, we have $\mathfrak{B}_{\mathfrak{E}}^{\phi, b}(\beta, 0)=\mathfrak{B}_{\mathfrak{E}}^{\phi, b}(\beta)$ and $f \in \mathfrak{B}_{\mathfrak{E}}^{\phi, b}(\beta)$ if the following conditions are satisfied:

$$
\left|\arg \left[\frac{z\left[\left(\mathfrak{D}^{b} f(z)\right)^{\prime}\right]^{\phi}}{\mathfrak{D}^{b} f(z)}\right]\right|<\frac{\beta \pi}{2} \quad z \in \nabla,
$$

and

$$
\left|\arg \left[\frac{h\left[\left(\mathfrak{D}^{b} g(h)\right)^{\prime}\right]^{\phi}}{\mathfrak{D}^{b} g(h)}\right]\right|<\frac{\beta \pi}{2} \quad h \in \nabla,
$$

where $f(z) \in \mathfrak{E}, \phi \geq 1,0<\beta \leq 1$ and

$$
g(h)=h-m_{2} h^{2}+\left(2 m_{2}^{2}-m_{3}\right) h^{3}-\left(5 m_{2}^{3}-5 m_{2} m_{3}+m_{4}\right) h^{4}+\cdots
$$

We note that for $b=0, \phi=1$ and $\psi=0$ the class $\mathfrak{B}_{\mathfrak{E}}^{1,0}(\beta, 0)=S_{\mathfrak{E}}^{*}(\beta)$ is class of strongly bi-starlike functions of order $\beta(0<\beta \leq 1)$. When $b=1, \phi=1$ and $\psi=0$ the class $\mathfrak{B}_{\mathfrak{E}}^{1,1}(\beta, 0)=\mathcal{K}_{\mathfrak{E}}^{*}(\beta)$ is class of strongly bi-convex functions of order $\beta(0<\beta \leq 1)$.

Remark 2.2. For $b=0$ we have class introduced and studied in [8].

Now we have the following theorem and the proof.

Theorem 2.1. Let $f(z)$ given by $(1.1)$ be in the class $\mathfrak{B}_{\mathfrak{E}}^{\phi, b}(\beta, \psi)$. Then

$$
\left|m_{2}\right| \leq \frac{2 \beta}{\sqrt{3^{b} \beta(6 \phi-4 \psi-2)+2^{2 b}\left[2 \beta\left(2 \phi^{2}-4 \phi+\psi^{2}+2 \phi \psi-2 \psi+1\right)\right.}}
$$

and

$$
\left|m_{3}\right| \leq \frac{4 \beta^{2}}{2^{2 b}(2 \phi-\psi-1)^{2}}+\frac{2 \beta}{3^{b}(3 \phi-2 \psi-1)}
$$


Proof. It follows from (2.1) and (2.2) that

$$
\frac{z\left[\left(\mathfrak{D}^{b} f(z)\right)^{\prime}\right]^{\phi}}{(1-\psi) \mathfrak{D}^{b} f(z)+\psi \mathfrak{D}^{b+1} f(z)}=[y(z)]^{\beta}
$$

and

$$
\frac{h\left[\left(\mathfrak{D}^{b} g(h)\right)^{\prime}\right]^{\phi}}{(1-\psi) \mathfrak{D}^{b} g(h)+\psi \mathfrak{D}^{b+1} g(h)}=[x(h)]^{\beta}
$$

where $y(z)$ and $x(u)$ are in the class $\mathcal{P}$ which is of the form

$$
y(z)=1+y_{1} z+y_{2} z^{2}+y_{3} z^{3}+\cdots
$$

$$
x(h)=1+x_{1} h+x_{2} h^{2}+x_{3} h^{3}+\cdots
$$

Hence,

$$
\begin{gathered}
{[y(z)]^{\beta}=1+\beta y_{1} z+\left(\beta y_{2}+\frac{\beta(\beta-1) y_{1}^{2}}{2 !}\right) z^{2}+\cdots} \\
{[x(h)]^{\beta}=1+\beta x_{1} h+\left(\beta x_{2}+\frac{\beta(\beta-1) x_{1}^{2}}{2 !}\right) h^{2}+\cdots .}
\end{gathered}
$$

Now, equating the coefficient in (2.7) and (2.8) we get

$$
(2 \phi-\psi-1) 2^{b} m_{2}=\beta y_{1}
$$

$$
\begin{gathered}
2^{2 b}\left(2 \phi^{2}-4 \phi+\psi^{2}+2 \phi \psi-2 \psi+1\right) m_{2}^{2}+3^{b}(3 \phi-2 \psi-1) m_{3}=\beta y_{2}+\frac{\beta(\beta-1) y_{1}^{2}}{2 !}, \\
-(2 \phi-\psi-1) 2^{b} m_{2}=\beta x_{1}
\end{gathered}
$$

$$
\begin{aligned}
3^{b}\left(2 m_{2}^{2}-m_{3}\right)(3 \phi-2 \psi-1)+\left(2 \phi^{2}-4 \phi+\psi^{2}+2 \phi \psi-2 \psi+1\right) & m_{2}^{2} 2^{2 b} \\
& =\beta x_{2}+\frac{\beta(\beta-1) x_{1}^{2}}{2 !}
\end{aligned}
$$

From (2.11) and (2.13) we obtain

$$
y_{1}=-x_{1}
$$

and

$$
2^{2 b+1}(2 \phi-\psi-1)^{2} m_{2}^{2}=\beta^{2}\left(y_{1}^{2}+x_{1}^{2}\right)
$$

Also from (2.12), (2.14) and (2.16), we have 


$$
\begin{array}{r}
m_{2}^{2}=\frac{\beta^{2}\left(y_{2}+x_{2}\right)}{3^{b} \beta(6 \phi-4 \psi-2)+2^{2 b+1} \beta\left(2 \phi^{2}-4 \phi+\psi^{2}+2 \phi \psi-2 \psi+1\right)} \\
-(\beta-1) 2^{2 b}(2 \phi-\psi-1)^{2} \\
m_{2}^{2}=\frac{\beta^{2}\left(y_{2}+x_{2}\right)}{3^{b} \beta(6 \phi-4 \psi-2)+2^{2 b}\left[2 \beta\left(2 \phi^{2}-4 \phi+\psi^{2}+2 \phi \psi-2 \psi+1\right)\right.} \\
\left.-(\beta-1)(2 \phi-\psi-1)^{2}\right]
\end{array}
$$

Applying Lemma (1.1) for the coefficients $y_{2}$ and $x_{2}$, we get

$$
\left|m_{2}\right| \leq \frac{2 \beta}{\sqrt{3^{b} \beta(6 \phi-4 \psi-2)+2^{2 b}\left[2 \beta\left(2 \phi^{2}-4 \phi+\psi^{2}+2 \phi \psi-2 \psi+1\right)\right.}} .
$$

Which gives us the desired estimate on $\left|m_{2}\right|$ as asserted in (2.5).

Next, in order to find the bounds on $\left|m_{3}\right|$, subtracting (2.14) from (2.12) we get

$$
3^{b}(6 \phi-4 \psi-2) m_{3}-3^{b}(6 \phi-4 \psi-2) m_{2}^{2}=\beta\left(y_{2}-x_{2}\right)+\frac{\beta(\beta-1)}{2 !}\left(y_{1}^{2}-x_{1}^{2}\right)
$$

It follows from (2.15), (2.16) and (2.17) that

$$
m_{3}=\frac{\beta^{2}\left(y_{1}^{2}+x_{1}^{2}\right)}{2^{2 b+1}(2 \phi-\psi-1)^{2}}+\frac{\beta\left(y_{2}-x_{2}\right)}{3^{b}(6 \phi-4 \psi-2)}
$$

Applying Lemma 1.1 for the coefficients $y_{1}, y_{2}, x_{1}$ and $x_{2}$, we have

$$
\left|m_{3}\right| \leq \frac{4 \beta^{2}}{2^{2 b}(2 \phi-\psi-1)^{2}}+\frac{2 \beta}{3^{b}(3 \phi-2 \psi-1)}
$$

We get the desired estimate $\left|m_{3}\right|$ as asserted in (2.6).

Putting $\phi=1$ in Theorem 2.1, we have the following corollary.

Corollary 2.1. Let $f(z)$ given by $(1.1)$ be in the class $\mathfrak{B}_{\mathfrak{E}}^{1, b}(\beta, \psi)$. Then

$$
\left|m_{2}\right| \leq \frac{2 \beta}{\sqrt{4 \beta(1-\psi) 3^{b}+2^{2 b}\left[2 \beta\left(\psi^{2}-1\right)-(\beta-1)(1-\psi)^{2}\right]}}
$$

and

$$
\left|m_{3}\right| \leq \frac{4 \beta^{2}}{2^{2 b}(1-\psi)^{2}}+\frac{\beta}{3^{b}(1-\psi)}
$$

which is the results obtain by Jothibasu [9].

Putting $\psi=0$ in Corollary (2.1), we have the following corollary. 
Corollary 2.2. Let $f(z)$ given by $(1.1)$ be in the class $\mathfrak{B}_{\mathfrak{E}}^{b}(\beta, 0)$. Then

$$
\left|m_{2}\right| \leq \frac{2 \beta}{\sqrt{4 \beta 3^{b}+2^{2 b}(1-3 \beta)}}
$$

and

$$
\left|m_{3}\right| \leq \frac{4 \beta^{2}}{2^{2 b}}+\frac{\beta}{3^{b}}
$$

Now putting $b=0$ in Corollary (2.2), we obtain the coefficient estimate for well-known class $\mathfrak{B}_{\mathfrak{E}}^{0}(\beta, 0)=S_{\mathfrak{E}}^{*}(\beta)$ of strongly bi-starlike functions of order $\beta$ as in [5]. Also when $b=1$ in Corollary (2.2), we obtain well-known class $\mathfrak{B}_{\mathfrak{E}}^{1}(\beta, 0)=\mathcal{K}_{\mathfrak{E}}(\beta)$ of strongly bi-convex function of order $\beta$ and have the same results in [5].

\section{Coefficient Bounds for the Function Class $\mathfrak{B}_{\mathfrak{E}}^{\phi, b}(\mu, \psi)$}

Definition 3.1. A function $f(z)$ given by (1.1) is said to be in the class $\mathfrak{B}_{\mathfrak{E}}^{\phi, b}(\mu, \psi)$ if the following conditions are satisfied:

$$
\Re\left[\frac{z\left[\left(\mathfrak{D}^{b} f(z)\right)^{\prime}\right]^{\phi}}{(1-\psi) \mathfrak{D}^{b} f(z)+\psi \mathfrak{D}^{b+1} f(z)}\right]>\mu \quad z \in \nabla,
$$

and

$$
\Re\left[\frac{h\left[\left(\mathfrak{D}^{b} g(h)\right)^{\prime}\right]^{\phi}}{(1-\psi) \mathfrak{D}^{b} g(h)+\psi \mathfrak{D}^{b+1} g(h)}\right]>\mu \quad h \in \nabla,
$$

where $f(z) \in \mathfrak{E}, \phi \geq 1,0 \leq \mu<1,0 \leq \psi<1$ and

$$
g(h)=h-m_{2} h^{2}+\left(2 m_{2}^{2}-m_{3}\right) h^{3}-\left(5 m_{2}^{3}-5 m_{2} m_{3}+m_{4}\right) h^{4}+\cdots .
$$

Remark 3.1. Taking $\psi=0$ in the class $\mathfrak{B}_{\mathfrak{E}}^{\phi, b}(\mu, \psi)$, we have $\mathfrak{B}_{\mathfrak{E}}^{\phi, b}(\mu, 0)=\mathfrak{B}_{\mathfrak{E}}^{\phi, b}(\mu)$ and $f \in \mathfrak{B}_{\mathfrak{E}}^{\phi, b}(\mu)$ if the following conditions are satisfied:

$$
\Re\left[\frac{z\left[\left(\mathfrak{D}^{b} f(z)\right)^{\prime}\right]^{\phi}}{\mathfrak{D}^{b} f(z)}\right]>\mu \quad z \in \nabla,
$$

and

$$
\Re\left[\frac{h\left[\left(\mathfrak{D}^{b} g(h)\right)^{\prime}\right]^{\phi}}{\mathfrak{D}^{b} g(h)}\right]>\mu \quad h \in \nabla,
$$

where $f(z) \in \mathfrak{E}, \phi \geq 1,0 \leq \mu<1$ and

$$
g(h)=h-m_{2} h^{2}+\left(2 m_{2}^{2}-m_{3}\right) h^{3}-\left(5 m_{2}^{3}-5 m_{2} m_{3}+m_{4}\right) h^{4}+\cdots .
$$

We note that for $b=0, \phi=1$ and $\psi=0$ the class $\mathfrak{B}_{\mathfrak{E}}^{1,0}(\mu, 0)=S_{\mathfrak{E}}^{*}(\mu)$ is class of strongly bi-starlike functions of order $\mu(0 \leq \mu<1)$. When $b=1, \phi-1=\psi=0$ and the class $\mathfrak{B}_{\mathfrak{E}}^{1,1}(\mu, 0)=\mathcal{K}_{\mathfrak{E}}^{*}(\mu)$ is class of strongly bi-convex functions of order $\mu(0 \leq \mu<1)$. 
Remark 3.2. For $b=0$ we have class introduced and studied in [8].

Now we have the following theorem and the proof.

Theorem 3.1. Let $f(z)$ given by (1.1) be in the class $\mathfrak{B}_{\mathfrak{E}}^{\phi, b}(\mu, \psi)$. Then

$$
\left|m_{2}\right| \leq \sqrt{\frac{2(1-\mu)}{2^{2 b}\left(2 \phi^{2}-4 \phi+\psi^{2}+2 \phi \psi-2 \psi+1\right)+(3 \phi-2 \psi-1) 3^{b}}}
$$

and

$$
\left|m_{3}\right| \leq \frac{4(1-\mu)^{2}}{2^{2 b}(2 \phi-\psi-1)^{2}}+\frac{2(1-\mu)}{3^{b}(3 \phi-2 \psi-1)} .
$$

Proof. It follows from (3.3) and (3.4) that there exist $y, x \in \mathcal{P}$ such that

$$
\frac{z\left[\left(\mathfrak{D}^{b} f(z)\right)^{\prime}\right]^{\phi}}{(1-\psi) \mathfrak{D}^{b} f(z)+\psi \mathfrak{D}^{b+1} f(z)}=\mu+(1-\mu) y(z)
$$

and

$$
\frac{h\left[\left(\mathfrak{D}^{b} g(h)\right)^{\prime}\right]^{\phi}}{(1-\psi) \mathfrak{D}^{b} g(h)+\psi \mathfrak{D}^{b+1} g(h)}=\mu+(1-\mu) x(h)
$$

where $y(z)$ and $x(h)$ in $\mathcal{P}$ given by (2.9) and (2.10), that is

$$
\mu+(1-\mu) y(z)=1+(1-\mu) y_{1} z+(1-\mu) y_{2} z^{2}+\cdots
$$

and

$$
\mu+(1-\mu) x(h)=1+(1-\mu) x_{1} h+(1-\phi) x_{2} h^{2}+\cdots
$$

Equating the coefficients of (3.7) and (3.8) we get

$$
\begin{gathered}
2^{2 b}\left(2 \phi^{2}-4 \phi+\psi^{2}+2 \phi \psi-2 \psi+1\right) m_{2}^{2}+3^{b}(3 \phi-2 \psi-1) m_{3}=(1-\mu) y_{2} \\
-(2 \phi-\psi-1) 2^{b} m_{2}=(1-\mu) x_{1} \\
3^{b}\left(2 m_{2}^{2}-m_{3}\right)(3 \phi-2 \psi-1)+\left(2 \phi^{2}-4 \phi+\psi^{2}+2 \phi \psi-2 \psi+1\right) m_{2}^{2} 2^{2 b}=(1-\mu) x_{2} .
\end{gathered}
$$

From (3.9) and (3.11) we get

$$
y_{1}=-x_{1}
$$


and

$$
2^{2 b+1}(2 \phi-\psi-1)^{2} m_{2}^{2}=(1-\mu)^{2}\left(y_{1}^{2}+x_{1}^{2}\right)
$$

Now adding (3.10), (3.12) and (3.14), we deduce that

$$
\begin{gathered}
m_{2}^{2}=\frac{(1-\mu)\left(y_{2}+x_{2}\right)}{2^{2 b+1}\left(2 \phi^{2}-4 \phi+\psi^{2}+2 \phi \psi-2 \psi+1\right)+2(3 \phi-2 \psi-1) 3^{b}} \\
\left|m_{2}^{2}\right| \leq \frac{(1-\mu)\left(\left|y_{2}\right|+\left|x_{2}\right|\right)}{2^{2 b+1}\left(2 \phi^{2}-4 \phi+\psi^{2}+2 \phi \psi-2 \psi+1\right)+2(3 \phi-2 \psi-1) 3^{b}}
\end{gathered}
$$

Applying Lemma 1.1 for the coefficients $y_{2}$ and $x_{2}$, we have

$$
\left|m_{2}\right| \leq \sqrt{\frac{2(1-\mu)}{2^{2 b}\left(2 \phi^{2}-4 \phi+\psi^{2}+2 \phi \psi-2 \psi+1\right)+(3 \phi-2 \psi-1) 3^{b}}}
$$

which gives us the desired estimate on $\left|m_{2}\right|$ as asserted in (3.5).

Hence in order to get the bound on $\left|m_{3}\right|$, by subtracting (3.12) from (3.10), we get

$$
3^{b}(6 \phi-4 \psi-2) m_{3}-3^{b}(6 \phi-4 \psi-2) m_{2}^{2}=(1-\mu)\left(y_{2}-x_{2}\right)
$$

$$
m_{3}=m_{2}^{2}+\frac{(1-\mu)\left(y_{2}-x_{2}\right)}{3^{b}(6 \phi-4 \psi-2)}
$$

then from (3.14), we have

$$
m_{3}=\frac{(1-\mu)^{2}\left(y_{1}^{2}+x_{1}^{2}\right)}{2^{2 b+1}(2 \phi-\psi-1)^{2}}+\frac{(1-\mu)\left(y_{2}-x_{2}\right)}{3^{b}(6 \phi-4 \psi-2)}
$$

Applying Lemma 1.1 for the coefficients $y_{1}, y_{2}, x_{1}$ and $x_{2}$, we have

$$
\left|m_{3}\right| \leq \frac{4(1-\mu)^{2}}{2^{2 b}(2 \phi-\psi-1)^{2}}+\frac{2(1-\mu)}{3^{b}(3 \phi-2 \psi-1)} .
$$

We get desired estimate on $\left|m_{3}\right|$ as asserted in (3.6).

Putting $\phi=1$ in Theorem 3.1, we have the following corollary.

Corollary 3.1. Let $f(z)$ given by $(1.1)$ be in the class $\mathfrak{B}_{\mathfrak{E}}^{1, b}(\mu, \psi)$. Then

$$
\left|m_{2}\right| \leq \sqrt{\frac{2(1-\mu)}{2^{2 b}\left(\psi^{2}-1\right)+2(1-\psi) 3^{b}}}
$$

and

$$
\left|m_{3}\right| \leq \frac{4(1-\mu)^{2}}{2^{2 b}(1-\psi)^{2}}+\frac{2(1-\mu)}{3^{b}(1-\psi)}
$$


which is the results obtain by Jothibasu [9].

Putting $\psi=0$ in Corollary (3.1), we have the following corollary.

Corollary 3.2. Let $f(z)$ given by $(1.1)$ be in the class $\mathfrak{B}_{\mathfrak{E}}^{b}(\mu, 0)$. Then

$$
\left|m_{2}\right| \leq \sqrt{\frac{1-\mu}{3^{b}-2^{2 b-1}}}
$$

and

$$
\left|m_{3}\right| \leq \frac{4(1-\mu)^{2}}{2^{2 b}}+\frac{2(1-\mu)}{3^{b}}
$$

Now putting $b=0$ in Corollary (3.2), we obtain the coefficient estimate for well-known class $\mathfrak{B}_{\mathfrak{E}}^{0}(\mu, 0)=S_{\mathfrak{E}}^{*}(\mu)$ of bi-starlike functions of order $\mu$ as in [5]. Also when $b=1$ in Corollary (3.2), we obtain well-known class $\mathfrak{B}_{\mathfrak{E}}^{1}(\mu, 0)=\mathcal{K}_{\mathfrak{E}}(\mu)$ of bi-convex function of order $\mu$ and have the same results in [5].

Remark 3.3. When $b=0$, the results acquired in this paper corresponds with the results considered in [8]. Also, for the different pick of b the results considered in this paper would pilot to many known and new results.

Acknowledgment. The author wish to thank the referees(s) for their relevant suggestions which improved the presentation of the paper.

\section{REFERENCES}

[1] A. Aldawish, T. Al-Hawary and B.A. Frasin, Subclasses of bi-univalent function defined by Frasin differential operator, Mathematics. 8 (2020), 783.

[2] K.O. Babalola, On $\lambda$-pseudo-starlike function, J. Class. Anal. 3 (2013), 137-147.

[3] D.A. Brannan, J.G. Clunie (Eds.), Aspects of Contemporary Complex Analysis (Proceedings of the NATO Advanced Study Institute held at the University of Durham, Durham; July 1-20, 1979), Academic Press, New York and London, 1980.

[4] D.A. Brannan, J. Clunie, W.E. Kirwan, Coefficient estimates for a class of star-like functions, Canad. J. Math. 22 (1970), 476-485.

[5] D.A. Brannan, T.S. Taha, On some classes of bi-univalent functions, in: S.M. Mazhar, A. Hamoui, N.S. Faour (Eds.), Mathematical Analysis and Its Applications, Kuwait; February 18-21, 1985, in: KFAS Proceedings Series, vol. 3, Pergamon Press (Elsevier Science Limited), Oxford, 1988, pp. 53-60; see also Studia Univ. Babe-Bolyai Math. 31, no. 2, 70-77, 1986.

[6] P.L. Duren, Univalent functions, Grundlehren der Mathematischen Wissenschaften, Springer, New York, (2004).

[7] B.A. Frasin, and M.K. Aouf, New subclasses of bi-univalent functions, Appl. Math. Lett. 24 (2011), pp. 1569-1573. 
[8] S.B. Joshi, and P.P. Yadav, Coefficient bounds for new subclasses of bi-univalent function associated with pseudo-starlike functions, Ganita J. 69 (2019), 67-74.

[9] J. Jothibasu, Certain subclasses of bi-univalent functions defined by salagean operator, Elec. J. Math. Anal. Appl. 3 (2015), 150-157.

[10] M. Lewin, On a coefficients problem of bi-univalent functions, Proc. Amer. Math. Soc. 18 (1967), 63-68.

[11] G. Murugusundaramoorthy, N. Magesh and V. Prameela, Coefficient bounds for certain subclasses of bi-univalent functions, Abs. Appl. Anal. 2013 (2013), 573017.

[12] E. Netanyahu, The minimal distance of the image boundary from the origin and the second coefficient of univalent function in $|z|<1$, Proc. Arch. Ration. Mech. Anal. 32 (1969), 100-112.

[13] S.0. Olatunji, and P.T. Ajayi, On subclasses of bi-univalent functions of Bazelevic type involving linear salagean operator, Int. J. Pure. Appl. Math. 92 (2014), 645-656.

[14] A. B. Patil and U. H. Naik, Bounds on initial coefficients for a new subclass of bi-univalent functions, New Trends Math. Sci. 6 (1) (2018), 85-90.

[15] C.H. Pommerenke, Univalent Functions, Vandendoeck and Rupercht, Gottingen, (1975).

[16] G.S. Salagean, Subclasses of Univalent functions, Lecture Notes in Math., Spinger Verlag, Berlin., 1013 (1983), 362-372.

[17] T.G. Shaba, On some new subclass of bi-univalent functions associated with Opoola differential operator, Open J. Math. Anal. 4(2) (2020), 74-79.

[18] T. G. Shaba, Certain new subclasses of analytic and bi-univalent functions using salagean operator, Asia Pac. J. Math. 7 (2020), 29.

[19] T. G. Shaba, Subclass of bi-univalent functions satisfying subordinate conditions defined by Frasin differential operator, Turk. J. Inequal. 4(2) (2020), 50-58.

[20] H.M. Srivastava and S. Owa (eds), Current topics in analytic function theory, World Sci. Publ., 1992.

[21] T.S. Taha, Topics in univalent functions theory, Ph.D. Thesis, University of London, London, UK, 1998.

[22] Q.H. Xu, Y.C. Gui and H.M. Srivastava, A certain general subclass of analytic and bi-univalent functions and associated coefficient estimate problems, Appl. Math. Comput. 218 (2012), 11461-11465. 